\title{
Dynamics of multicomponent, multifield quintessence
}

\author{
Tzihong Chiueh \\ Department of Physics, National Taiwan University, Taipei, Taiwan \\ and Institute of Astronomy and Astrophysics, Academia Sinica, Taipei, Taiwan
}

(Received 7 February 2002; published 24 May 2002)

\begin{abstract}
The self-consistent dynamics of the multicomponent, $N$-field quintessence and gravity is formulated as relativistic $\mathrm{N}$-body dynamics, embedded in a static viscous flat space and under the forces given by the interacting Lorentz scalar potential via exchange of field bosons. The Ratra-Peebles power-law potential of effective single-field quintessence can be derived from this "microscopic" perspective. In certain situations, the effective dynamics can be made identical to that of single complex quintessence, except that the overall $U(1)$ symmetry is not manifestly broken. The present formulation provides a convenient gauge for analyzing superhorizon perturbations and possibly for quantization of superhorizon fields and gravity together.
\end{abstract}

DOI: 10.1103/PhysRevD.65.123502

PACS number(s): 98.80.Cq, 95.30.Sf, 98.80.Es

\section{INTRODUCTION}

The discovery of accelerating Hubble expansion has inspired intense investigations on various acceleration mechanisms [1-3]. Conventionally, the cosmological constant of a suitable value does just fine to explain satisfactorily all existing data [4-6]. However, as the quality of cosmological observations are rapidly to be further improved in the near future, it is therefore timely to explore possibilities beyond what has conventionally been taken for granted. The proposal of quintessence marks an important breakthrough in how one perceives the major constituents of the present-day universe, which have conventionally been believed to be describable by classical physics. This new constituent is a field that permeates the entire space in a relative uniform manner, and is not a collection of matter particles. Investigations on the quintessence dynamics and its ultimate cosmological implications have been an active research area in recent years [7].

The field description in the context of cosmology is not new, but was in the past limited to the physics of the very early universe, where classical physics no longer holds. The inflaton field that drives early-universe inflation is a pronounced example. Reheating is another example; it addresses how the radiation is created by dissipating the rapid oscillation of the same inflaton field previously driving the inflation $[8,9]$. In fact, the proposal of quintessence is well motivated by the recognition that the origin of the cosmological constant can be the ground state of a certain scalar field. If so, it is not too far-fetched to consider the field to be now evolving on its way to settle to the ground state on the cosmological time scale.

Somewhat different from the pure scalar field dynamics in the very early universe, the dynamics of quintessence must generally take into account other evolving energy components, such as matter and radiation, in the universe. The presence of other energy components turns out to dilute the strength of both the "drag" resulting from Hubble expansion and the "force" given by the quintessence potential. It is therefore of relevance to investigate how all coupled energy components, including gravity, evolve in time under a unified framework. This paper first aims to address this issue.

On the other hand, there may be more than one quintessence field present in the universe. In general, these $N$ fields couple through some mutual interactions, in addition to gravity. The complexity of the multifield problem rises drastically, due partly to the increasing number of $N$ degrees of freedom, and also to the $N^{2}$ mutual interactions. Nevertheless, when the number of fields $N$ is sufficiently large the problem can become simple again, since one may extract a few relevant dynamical variables and derives an effective theory from this many-field problem. In an analogy to manybody problems, such an effective theory is derived from a field-theoretical approach. If this program can be made successful, it will provide a good foundation for the effective potential of the effective quintessence in terms of elemental mutual interactions of individual quintessence fields. Furthermore, the mutual interactions should be regarded as exchanges of some mediate bosons in the field space. The present paper mainly aims at formulating a useful framework for such a many-field problem.

It also turns out the present formulation, using $T$ $[\equiv \ln (a)]$ as the new time variable, is most convenient for analyzing cosmological perturbations in the long-wavelength limit [10]. In this gauge $T$ can remain unperturbed to the order of $k / H$, the ratio of the Hubble radius to the wavelength of superhorizon perturbation, and the cosmological perturbations can be conveniently perceived as the evolving difference of two homogeneous and isotropic universes with slightly different initial conditions.

In this paper we do not wish to distinguish the quintessence from the scalar fields that were active in the early universe before photons were produced (reheating), and we regard these fields altogether as the quintessence. That is, one may turn off the photon fluid in the present formulation to address the inflaton dynamics. The dynamics of the single quintessence field is formulated in Sec. II. Extension to multicomponent, multifields is presented in Sec. III, where ex- 
amples for deriving the effective quintessence models are given. We also touch upon the cosmological perturbations with the present formulation in Sec. IV. Conclusions and comments are given in Sec. V.

\section{SINGLE-FIELD QUINTESSENCE}

Assuming a homogeneous and isotropic universe, we have the equation of motion for the scalar field $\phi$ under a potential $V(\phi)$ as

$$
\ddot{\phi}+3 H \dot{\phi}+\frac{\partial V(\phi)}{\partial \phi}=0 .
$$

The Einstein equations read

$$
\begin{aligned}
& H^{2} \equiv\left(\frac{\dot{a}}{a}\right)^{2}=\frac{2 g V_{e f f}}{1-g \phi^{\prime 2}}, \\
& \frac{\ddot{a}}{a}=-2 H^{2}+3 g\left[2 V_{e f f}-\left(\rho_{f}+p_{f}\right)\right],
\end{aligned}
$$

where $g \equiv 4 \pi G / 3, \phi^{\prime} \equiv d \phi / d T$ with $T \equiv \ln a, V_{e f f} \equiv V(\phi)$ $+\rho_{f}$, and $\rho_{f}$ and $p_{f}$ are the energy density and pressure of other fluid components, which may also include the space curvature. The space curvature can generally be regarded as a fluid in this homogeneous and isotropic limit, with an equation of state $p_{f}=-\rho_{f} / 3$. The time dependence of $\rho_{f}$ is proportional to $a^{-2}$ and its value can be either positive or negative depending on whether the space has negative or positive curvature, respectively.

These coupled equations can be simplified if we let the independent variable of Eq. (1) be T. Thus, $\dot{\phi}=H \phi^{\prime}$ and $\ddot{\phi}$ $=H^{2} \phi^{\prime \prime}+\dot{H} \phi^{\prime}$, with $H$ and $\dot{H}$ replaced by Eq. (2). Equations (1) and (2) combine to yield a single equation:

$$
\phi^{\prime \prime}+\left(1-g \phi^{\prime 2}\right)\left[3\left(1-\frac{\rho_{f}+p_{f}}{2 V_{e f f}}\right) \phi^{\prime}+\frac{1}{2 g} \frac{\partial \ln V_{e f f}}{\partial \phi}\right]=0 .
$$

The peculiar feature of this equation is that $\sqrt{g} \phi^{\prime}= \pm 1$ are the fixed points, and solutions with $g \phi^{\prime 2}-1<0$ or $>0$ cannot cross the fixed points to change the signs. The peculiar behavior at the fixed points means that there exists a maximum rate of coherent change of $\phi$ over the horizon. This condition is also reflected in the expression of $H^{2}$ in Eq. (2), where $H^{2}$ may become singular. If $V_{\text {eff }}$ does not change sign, the singularities in $H^{2}$ cannot be removed by having the numerator to vanish simultaneously with the denominator. Such an unphysical result is automatically avoided in Eq. (3), demanding that $g \phi^{\prime 2}-1$ must remain of the same sign. As a non-negative $V_{\text {eff }}$ is of physical relevance, we shall focus on the causal regime where $g \phi^{\prime 2}<1$ in the rest of this paper.

Having understood so, we may make an analogy of the field dynamics to particle dynamics, in that one identifies $\sqrt{g} \phi^{\prime}$ as the velocity $v,\left(1-g \phi^{\prime 2}\right)^{-1 / 2}$ as the Lorentz factor $\gamma$, and $u=\gamma \sqrt{g} \phi^{\prime}$ as the momentum. This nonlinear transformation puts Eq. (3) into

$$
\frac{d u}{d T}+3\left(1-\frac{\rho_{f}+p_{f}}{2 V_{e f f}}\right) u+\frac{\gamma}{2 \sqrt{g}} \frac{\partial \ln V_{e f f}}{\partial \phi}=0 .
$$

The first and third terms are the " 01 " components of a second-rank tensor and the second term is a vector. It demonstrates that this dynamical system is not Lorentz covariant, because the frictional force given by the Hubble expansion behaves as if the system contains a viscous medium which has already chosen a preferred reference frame.

Of particular interest in Eqs. (3) or (4) is the softening effect to the field $\phi$ provided by another energy component $\rho_{f}$. When the field has a vacuum state $\phi_{v}$, where $V\left(\phi_{v}\right)$ $=V^{\prime}\left(\phi_{v}\right)=0$, the "force" $-\partial \ln V_{\text {eff }} / \partial \phi$ can be locally expanded as $-2\left(\phi-\phi_{\min }\right)\left[\left(\phi-\phi_{\min }\right)^{2}+\left(2 \rho_{f} / V^{\prime \prime}\right)\right]^{-1}$. It is analogous to a two-dimensional Coulomb attractive force given by a finite-sized charged distribution. If the soft core does not exist, i.e., $\rho_{f}=0$, the "kinetic energy," or $\gamma$, will become infinity at the vacuum, so that $H^{2}$ in Eq. (2) remains finite.

To put the above into a quantitative perspective, we note that $g^{-1 / 2} \sim E_{\text {planck }}$, the Planck energy. Hence the dimensionless "displacement" $\sqrt{g} \phi$ and velocity $\sqrt{g} \phi$ ' are all normalized to the Planck scale. The Planck scale thus typically implies a "relativistic" regime. However, the reverse statement is typically not true. The energy scale much below the Planck regime can also be "relativistic," as long as the dynamical time scale is sufficiently small so that $d / d T$ is large despite $\phi$ being small. Such a situation is commonly encountered when $V_{\text {eff }} \rightarrow 0$.

Despite not being Lorentz covariant, the dynamics can still be put in a Lagrangian framework that describes the self-consistent evolution of field and gravity. A straightforward inspection shows that the action for the coupled dynamics becomes

$$
\int L d T=-\int e^{3 T} d T \gamma^{-1} \sqrt{2 g V_{e f f}}
$$

By defining a new potential $\Phi(\phi, T) \equiv \ln V_{e f f}+6 T$, we have here an action of particle dynamics in the presence of a dilation field: $\sqrt{2 g} \int \exp [\Phi(\phi, T) / 2] d \tau$, where $\Phi$ is a dilaton field and $\tau$ is the proper time. In fact, the action given in Eq. (5) can be derived rigorously by summing up the Lagrangians of the quintessence field, gravity, and other energy components, as will be described in the next section.

Although there is no obvious conservation law for $\phi$ due to cosmic expansion, this Lagrangian nevertheless allows us to obtain a Hamiltonian, which can yield an approximately conserved quantity. This Hamiltonian $h$ is constructed by the usual procedure

$$
h=\phi^{\prime}\left(\partial L / \partial \phi^{\prime}\right)-L=\gamma \sqrt{2 g V_{e f f}} e^{3 T} .
$$


Actually, $h=H \exp (3 T)$ according to Eq. (2).

To show $h$ to be indeed useful, we obtain the equation for $h$ by multiplying Eq. (5) by $u$ to get

$$
\frac{d \ln h}{d T}=\frac{3}{\gamma^{2}}\left(1-\frac{\rho_{f}+p_{f}}{2 V_{e f f}}\right),
$$

or after a straightforward algebra

$$
\frac{d}{d T}\left(V+u^{2} V_{e f f}\right)=-6 u^{2} V_{e f f}
$$

It is clear that Eq. (7) is appropriate for the "ultrarelativistic" regime, where the right-hand side can be ignored and one obtains approximate conserved quantities.

The "ultrarelativistic speed" means that $\sqrt{g} \phi$ ' $\rightarrow \pm 1$, implying $\sqrt{g} \phi \rightarrow \pm T$ in the large $|T|$ limit. The "Lorentz" factor $\gamma$ can stays large as long as $2 g V_{e f f} \ll h^{2} \exp (-6 T)$. On the other hand, since $h=\mathrm{const}$ the Hubble parameter $H$ $(=d T / d t)$ is proportional to $\exp (-3 T)$ in this regime. Solving for $T(t)[\equiv d \ln (a) / d \ln t]$, we find that $\exp (T) \sim a(t) \propto t^{1 / 3}$, i.e., a universal expansion regardless of the form of $V(\phi)$ in this kinetic-energy-dominated regime. This is the slowest asymptotic Hubble expansion rate one may hope to obtain.

From Eq. (4), one also sees that apart from the usual notion of inflation where a large range of flat $V(\phi)$ and a potential minimum are needed for it to occur, the inflation can also take place in a peculiar situation when $\rho_{f}=0$, the potential has a maximum, and the incoming kinetic energy is tuned to have just the right value for it to be completely consumed upon climbing to the potential top. Since $v=0$ and $\partial \ln V / \partial \phi=0$, a state situated exactly at the separatrix also gives a constant $H$, thereby yielding inflation. Before leaving this simplest single-field dynamics, we shall also look into the dynamics of typical relevant potentials.

\section{A. Exponential potential}

The exponential potential, $V(\phi)=\exp (6 \beta \sqrt{g} \phi)$, has interesting conformal symmetry, when $\rho_{f}=0$ and $\beta= \pm 1$. The conformal transformation, $\sqrt{g} \bar{\phi}=\left(1 \pm v_{t} / 1 \mp v_{t}\right)^{1 / 2} \gamma_{t}(\sqrt{g} \phi$ $\left.-v_{t} T\right)-T_{0} \quad$ and $\quad \bar{T}=\left(1 \pm v_{t} / 1 \mp v_{t}\right)^{1 / 2} \gamma_{t}\left(T-v_{t} \sqrt{g} \phi\right)$ $+T_{0}-(1 / 6) \ln \left(1 \pm v_{t} / 1 \mp v_{t}\right)$, transforms the original action $\quad \int d T \sqrt{1-g(d \phi / d T)^{2}} \exp [3( \pm \sqrt{g} \phi+T)]$ into $\int d \bar{T} \sqrt{1-g(d \bar{\phi} / d \bar{T})^{2}} \exp [3( \pm \sqrt{g} \bar{\phi}+\bar{T})]$, where the transform "velocity" $v_{t}$ and the shift $T_{0}$ are both constants. The transformed action assumes the same form as the original action, and thus yields the same equation of motion. In fact, this particular case $|\beta|=1$ lies at the boundary of two distinctly different regimes, to be discussed below.

When $|\beta|>1$, it is possible to make the above transformation such that the $\bar{T}$ dependence vanishes by choosing $v_{t}$ $=-\beta^{-1}$. This is a spacelike regime where the Lagrangian becomes time independent and free of friction. The conserved Hamiltonian $\bar{h}$ is a constant of motion and becomes $\bar{h} \propto \bar{\gamma} \exp [3 \sqrt{g} \bar{\phi}(\beta \pm 1)]$. It allows for a solution $\bar{\phi}$ $=[1 / 3 \sqrt{g}(\beta \pm 1)] \ln \left\{\cosh \left[3(\beta \pm 1)\left(\bar{T}-\bar{T}_{0}\right) / 2\right]\right\}$.
On the other hand, when $|\beta|<1$, one may choose $v_{t}=$ $-\beta$ so that the original exponential potential is transformed to become a $\phi$-independent vacuum energy, thereby yielding solutions that experience only the frictional force. This is a timelike regime. The Lagrangian becomes $\propto \bar{\gamma}^{-1} \exp [3(1$ $\pm \beta) \bar{T}]$. Since there is no applied force in the transformed coordinates, we have an asymptotic solution at rest, $\bar{\phi}=0$ and $d \bar{\phi} / d \bar{T}=0$. It then follows that $H \propto \gamma \exp (3 \beta \sqrt{g} \phi)$ $\propto \exp \left(3 \beta v_{t} T\right)=\exp \left(-3 \beta^{2} T\right)$. Hence, the scaling factor $a$ $\propto t^{1 / 3 \beta^{2}}$. For $\beta^{2}<1 / 3$, these solutions result in power-law inflation, for which the universe undergoes accelerating expansion.

\section{B. Power-law potential}

The repulsive power-law potentials, $V(\phi)=V_{0} \phi^{-n}$ for $n>0$, can also yield inflation-like solutions, known as the Ratra-Peebles potentials [11]. The inertia, i.e., the second derivative of Eqs. (3) or (4), can only be relevant only for a short while for the field to pick up the "speed." But it will quickly become subdominant, and the field reaches the terminal "velocity," where the frictional force balances the applied force. Moreover, as the terminal speed is always "nonrelativistic," Eqs. (3) or (4) give the solution, $\phi \propto T^{1 / 2}$, and hence $H \propto \sqrt{V} \propto T^{-n / 2}$, or $a \propto \exp \left(t^{2 /(2+n)}\right)$, also an inflationary solution. The above is for the case $\rho_{f}=0$. When $\rho_{f}$ is finite and even dominates $V_{e f f}$, the balance of friction and applied force then gives $V(\phi) \propto a^{-\kappa n / n+2}$, where we have let $\rho_{f}$ $\propto a^{-\kappa}$. Apparently $V$ declines more slowly than $\rho_{f}$ does, and hence the scalar field will eventually dominate and the Hubble expansion approaches that of the fluid-free case.

The dynamics can be more complex when the power-law potential is attractive, $V(\phi) \propto \phi^{2 n}$, as it exhibits anharmonic damped oscillations. Nevertheless, the following general property holds. First, since the attractive force, $-\partial \ln V_{e f f} / \partial \phi$, becomes increasingly stronger in the regime where the average potential strength $\langle V\rangle \gg \rho_{f}$, the oscillation frequency is increasingly higher. When the oscillation frequency is sufficiently high, one has a small number, the ratio of the oscillation period to the Hubble time, as an expansion parameter for a perturbative treatment in evaluating how the oscillation amplitude declines in time. (This high-frequency regime may also occur even when $\rho_{f} \gg\langle V\rangle$ because $\rho_{f}$ can decrease rapidly in time. An accurate assessment of when the high-frequency regime holds will be deferred to the end of this section.)

To the leading order, the orbit in one oscillation can be viewed as dissipationless, thereby allowing one to calculate the "ideal" orbit. One may subsequently substitute the "ideal" orbit into the damping term to evaluate how much damping it incurs to the orbit in one oscillation period. The long-term damping of the oscillation amplitude can therefore be evaluated to the leading order after these two steps.

Ignore the right-hand side of Eq. (8) and one gets the momentum of the dissipationless oscillation orbit:

$$
u^{2}=\frac{E(T)-V(\phi)}{V(\phi)+\rho_{f}(T)},
$$


where $E$ is a quasistatic constant of motion, slowly dissipated by the right-hand side of Eq. (8). When $\rho_{f} \geqslant\langle V\rangle$, the dynamics assumes nonrelativistic motion and hence $E \approx \rho_{f} v^{2}$ $+V(\phi)$. That is, quintenssence acquires an effective mass of $2 \rho_{f}$. Averaged over one oscillation period, Eq. (8) gives, to the leading order, the rate of secular change for $E$ :

$$
\begin{aligned}
\frac{D E}{D T} & \approx-6 \frac{\int d T\left(V+\rho_{f}\right) u^{2}}{\int d T} \\
& =-6 \frac{\int d \phi \sqrt{E-V(\phi)}}{\int d \phi(\sqrt{E-V(\phi)})^{-1}} \\
& =-3 \frac{d E}{d \ln \left(\int d \phi \sqrt{E-V(\phi)}\right)} .
\end{aligned}
$$

Here we have used the nonrelativistic approximation $d T$ $=\sqrt{g} d \phi v^{-1}, D / D T$ is the derivative on the slow time scale and the second equality is merely an algebraic equality. Since the right-hand side of the third equality is a derivative on the slow quantity, Eq. (10) can thus be trivially integrated to yield

$$
T(E)-T_{0}=-\frac{1}{3} \ln \left(\int d \phi \sqrt{E-V(\phi)}\right),
$$

where $T_{0}$ is also an integration constant.

We now apply the above results to the attractive powerlaw potential, $V(\phi) \propto \phi^{2 n}$. The integral $\int d \phi \sqrt{E-V(\phi)}$ $\propto E^{(n+1) / 2 n}$, and $T\left(V_{0}\right)=-\ln E[(n+1) / 6 n]+$ const, or $E$ $\propto a^{-6(n+1) / n}$. That is, a quadratic potential, $n=1$, yields $E$ $\propto a^{-3}$, similar to a matter fluid and a quartic potential, $n$ $=2$, gives $E \propto a^{-4}$, similar to a photon fluid.

Having Eq. (11), we may further evaluate $D t=\int d t$ $\left(=\int d T H^{-1}\right)$, so that the averaged Hubble expansion rate $\bar{H}=D T / D t$ has the expected expression

$$
\bar{H}=\frac{D T}{D t}=\sqrt{2 g[E(T)+\rho(T)]} .
$$

Equations (11) and (12) together permit the deceleration parameter to also be evaluated,

$$
q(T) \equiv \frac{1}{2 a \bar{H}^{2}} \frac{D^{2} a}{D t^{2}}=\frac{1}{2} \frac{D \ln \left[E(T)+\rho_{f}(T)\right]}{D T}+1
$$

All the above results for the long-term evolution are based on the assumption that oscillation frequency is much larger than the Hubble expansion rate. It is evident from Eqs. (3) or (4) that when $\rho_{f}$ is much greater than $V(\phi)$, the force strength is substantially reduced by the factor $V(\phi) / \rho_{f}$, thus reducing the oscillation frequency by $\sqrt{V(\phi) / \rho_{f}}$. Hence, there is a limit, beyond which the above results fail to hold. The regime in which this may happen is always "nonrelativistic," and one may approximate the right-hand side of Eq. (4) by $-\omega^{2} \phi$, where $\omega$ is the dimensionless oscillation frequency, to estimate $\omega \sim\left[(d V / d \phi) / 2 \sqrt{g} \phi\left(\rho_{f}+\phi\right)\right]^{1 / 2}$. Again, using the power-law potential, $V \propto \phi^{2 n}$, we find that $\omega$ $\propto \phi^{-1} \propto a^{3 /(n+1)}$ if $\langle V\rangle \gg \rho$, and that $\omega \propto \phi^{n-1} a^{3(1+w) / 2} \propto a^{b}$ when $\rho \gg\langle V\rangle$, where $b \equiv 3(1+w) / 2-3(n-1) /(n+1)$ and the fluid equation of state $w=p_{f} / \rho_{f}$ has been assumed. In the former case, all confining $(n>0)$ potentials give the desired high frequency. In the latter situation we, however, need $b \geqslant 0$, or $n \leqslant(3+w) /(1-w)$. For photon fluids, one needs $n \leqslant 5$, and for matter fluids, $n \leqslant 3$.

\section{DYNAMICS OF MULTICOMPONENT $N$ FIELDS}

\section{A. General framework}

The success in describing the field dynamics by relativistic particle dynamics motivates us to go one step further, and extend this formulation to the dynamics of multicomponent, multifields. Here, the component of a field refers to the requirement that it has a geometric structure as a vector.

As an illustration of how this can be done, we shall first consider a simple example of multicomponent, single-field quintessence with no fluid component present. In this case, the potential $V$ is a scalar and cannot be an arbitrary function of $\phi_{i}$, where $i$ is the component index. A particularly interesting potential is $V(\phi)=V_{0} \exp [\beta \cdot \phi]$, where $\beta$ satisfies $\beta^{2}=1$. In this particular case, the constant vector $\beta$ picks out a particular direction of $\phi$. Along this direction, one may perform the conformal Lorentz+shift transformation discussed in Sec. II for the single-component quintessence, and obtain an invariant action under such a transformation. The action is also invariant to the conformal helical (rotation + shift) transformation along the $\beta$ direction.

It turns out that the action of this particular $N$-component, single-field quintessence can also be the action of the $N$ $-M$ component, $M$-field quintessence. This is due to the fact that the Lorentz factor [cf. Eq. (5)] in the action contains the "velocities" of all fields, as will be elaborated below. Hence, the above conformal symmetry still holds for the $N-M$ component, $M$-field quintessence, as long as $g \Sigma_{i}\left(\phi_{i}^{\prime}\right)^{2}=1$.

The above example shows that when the potential $V$ assumes some particular forms, there can be no distinction between the multicomponent single-field and the multifield. To have a distinct difference, the potential should have a more general form. Below, we consider such a situation. For the purpose of illustration, we are confined to the twocomponent fields, which can be conveniently described by complex fields. Fields with more than two components can be easily generalized.

Let $\psi_{i}$ be the $i$ th of a system of $N$ two-component fields. We are interested in an autonomous system of interacting $N$ fields, where the interacting potential can be written as $V_{i, j}$ $=V\left(\left|\psi_{i}-\psi_{j}\right|\right)$ with $i \neq j$. The action for the system of $N$ fields, the gravitation and a fluid component can thus be written as 


$$
\begin{aligned}
S_{0}= & -l^{3} \int a^{3}(t) d t\left\{\left(\sum_{i} \frac{\dot{\psi}_{i}^{2}}{2}-\sum_{i, j} V_{i, j}\right)+\left(\frac{R}{12 g}\right)\right. \\
& \left.+\left[\lambda\left(\frac{n_{f}^{2} \dot{\theta}_{f}^{2}}{2\left(\rho_{f}+p_{f}\right)}-\frac{\rho_{f}+p_{f}}{2}\right)+p_{f}\right]\right\},
\end{aligned}
$$

where $l^{3}$ is the space volume, $R\left(\equiv(1 / 2)\left[(\dot{a} / a)^{2}+(\ddot{a} / a)\right]\right)$ the scalar curvature, $\lambda$ the Lagrangian multiplier, and $n_{f}$ and $\theta_{f}$ are the number density and velocity potential of the fluid, respectively.

The Lagrangian multiplier $\lambda$ is introduce to defined what the fluid 4-velocity $U_{\mu}$ is meant to be. In fact, a full fluid Lagrangian has been explored before [12], and here we consider only the spatially uniform limit. Variation of $S_{0}$ with respect to $\lambda$ gives the constraint

$$
\left(\frac{n_{f} \dot{\theta}_{f}}{\rho_{f}+p_{f}}\right)^{2}=1
$$

The variation with respect to $\theta_{f}$ yields a conservation law,

$$
\frac{a^{3} n_{f}^{2} \dot{\theta}_{f}}{\rho_{f}+p_{f}}=s^{2}
$$

where $s$ is a real constant. Since the 4-velocity satisfies $U_{\mu} U^{\mu}=1$, the constraint, Eq. (15), defines the fluid 4-velocity as $U_{\mu}=n_{f} \partial_{\mu} \theta_{f} /\left(\rho_{f}+p_{f}\right)$. It then follows that Eq. (16) gives $a^{3} n_{f} U_{0}=s^{2}$; as the fluid is at rest in the comoving frame of cosmic expansion, this becomes $a^{3} n_{f}=s^{2}$, i.e., the conservation of particle number density in a uniformly expanding fluid. Variation with respect to $n_{f}$ gives $\partial\left[\lambda \rho_{f}+(\lambda\right.$ $\left.-1) p_{f}\right] / \partial \ln n_{f}=\lambda\left(\rho_{f}+p_{f}\right)$. Note that the ideal-gas law demands $\partial \rho_{f} / \partial \ln n_{f}=\rho_{f}+p_{f}$, therefore fixing $\lambda=1$.

The Einstein equations are derived from this action by variation with respect to the scale factor $a$, and it gives, as before,

$$
\begin{aligned}
& H^{2}=g \frac{2 \sum_{i, j} V_{i, j}+\rho_{f}-p_{f}}{1-g \sum_{i}\left(\psi_{i}^{\prime}\right)^{2}-g\left[n_{f}^{2}\left(\theta_{f}^{\prime}\right)^{2} / \rho_{f}+p_{f}\right]}, \\
& \frac{\ddot{a}}{a}=-2 H^{2}+3 g\left(2 \sum_{i, j} V_{i, j}+\rho_{f}-p_{f}\right),
\end{aligned}
$$

where $\psi_{i}^{\prime} \equiv H^{-1} \dot{\psi}_{i}$ and $\theta_{f}^{\prime} \equiv H^{-1} \dot{\theta}_{f}$.

Now, replacing $d t$ by $d T / H$, substituting $H$ of Eq. (17) into $d / d T$, and recognizing $R=6 g\left(\rho_{\text {total }}-3 p_{\text {total }}\right)$, we finally obtain the effective $N$-field and fluid action for Eq. (14), much like how Eq. (5) is obtained. In this effective action the gravity is manifestly hidden:

$$
S_{0}=-l^{3} \int e^{3 T} d T(\bar{\Gamma})^{-1} \sqrt{g\left[2 \sum_{i, j} V_{i, j}+\rho_{f}-p_{f}\right]},
$$

where $(\bar{\Gamma})^{-1} \equiv \sqrt{1-g \sum_{i}\left(\psi_{i}^{\prime}\right)^{2}-g n_{f}^{2}\left(\theta_{f}^{\prime}\right)^{2} /\left(\rho_{f}+p_{f}\right)}$.

The action recovers Eqs. (15) and (16), and again the appropriate ideal-gas law is needed to fix $\lambda=1$. The nontrivial extension of this action from that of Eq. (5) is the appearance of an effective Lorentz factor $\bar{\Gamma}$, which is not the sum of individual Lorentz factors $\gamma_{i}$, as one may have expected. Rather, it is the sum of "kinetic energy" of all fields that contributes to $\bar{\Gamma}$. When $N$ is sufficiently large, it is obvious that $g\left(\psi_{i}^{\prime}\right)^{2} \ll 1$ in order for $\bar{\Gamma}$ to be real, thus demanding each individual field to be "nonrelativistic." In addition, it is straightforward to derive the corresponding Hamiltonian $h$, as we did for Eq. (6), and the relation $h=H \exp (3 T)$ still holds.

Although Eq. (18) contains a complete Lagrangian for describing the coupled dynamics of fluid and quintessence in terms of the logarithmic scaling factor $T$, the fluid and quintessence in fact form a master-slave system. That is, in terms of the variable $T$, the fluid dynamics is independent of the presence of quintessence, but the quintessence dynamics is governed by the presence of fluid. As the fluid dynamics can be easily solved when the equation of state is given, it is therefore possible to simplify the algebra by focusing on the quintessence piece of Eq. (18), with the fluid variables $\rho_{f}$ and $p_{f}$ treated as known functions of $T$. This is the strategy that was adopted in Sec. II and it gives a much simpler and practical result:

$$
S_{q}=-l^{3} \int e^{3 T} d T \Gamma^{-1} \sqrt{2 g V_{e f f}}
$$

with the fluid satisfying

$$
\frac{d \rho_{f}}{d T}=-3\left(\rho_{f}+p_{f}\right)
$$

where $\quad V_{e f f}=\sum_{i, j} V_{i, j}+\rho_{f}(T) \quad$ and $\quad \Gamma^{-1} \equiv \sqrt{1-g \sum_{i}\left(\psi_{i}^{\prime}\right)^{2}}$. Moreover, the Einstein equations, Eq. (17), now become Eq. (2), with the "Lorentz" factor in Eq. (2) replaced by the multifield $\Gamma$ defined here.

We would like to mention in passing that the way by which the multifield version of Eq. (3) is derived from this multifield quintessence action, Eq. (19), has a slight twist. Extend Eq. (3), $G=0$, to the expected multifield version, $G_{i}=0$. The Euler-Langrange equation of Eq. (19) in fact yields $\quad \sum_{j}\left[\left(\delta_{i j}-P_{i j}\right)+\Gamma^{-2} P_{i j}\right] G_{j}=0, \quad$ where $P_{i j} \equiv \delta_{i j}$ $-\psi_{i}^{\prime} \psi_{j}^{\prime} / \Sigma_{k}\left(\psi_{k}^{\prime}\right)^{2}$, a projection operator perpendicular to $\psi_{i}^{\prime}$. Since $\left(\delta_{i j}-P_{i j}\right)+\Gamma^{-2} P_{i j}$ is nonvanishing, this equation simply implies that the component of $G_{i}$ parallel to $\psi_{i}^{\prime}$ and those perpendicular to $\psi_{i}^{\prime}$ both vanish, and therefore $G_{i}=0$. The projection into two components relative to the "velocity" manifestly indicates that the special-relativistic effect is in action for producing anisotropic forces. In particular, Eq. (4) should be rewritten from $d u / d T=F$ to $d u_{i} / d T$ $=\Sigma_{j}\left[\left(\delta_{i j}-P_{i j}\right)+\Gamma^{-2} P_{i j}\right] F_{j}$ in the multifield case. This feature of dynamics given by Eq. (19) has been discussed extensively in the past, and the potential $V$ has been known as the Lorentz scalar potential [13]. 


\section{B. Coulomb field gas}

One may be contented with this Lagrangian, where $V_{i, j}$ is a given function. But one may also be somewhat more ambitious and ask whether there may be a fundamental dynamical equation that accounts for $V_{i, j}$. Actually, such a question is well motivated, since these $N$ fields are like $N$ particles. It is natural for $N$ particles to interact via the exchange of some mediate bosons, such as the familiar photons in electromagnetic interactions, and these mediate bosons obey their own dynamical equations. Here, we shall regard $V_{i, j}$ as the manifestation of such scalar mediate bosons of zero frequency. To attack this problem, we will need to go beyond the original discrete field $\psi_{i}$ and consider $V$ to be the field generated by and interacting with the discrete $\psi_{i}$, which in the continuum limit can be replaced by $\int d^{2} \psi \delta\left(\psi-\psi_{i}\right) \psi$ where the Dirac $\delta$ function is in use. The simplest version for $V(\psi)$ dynamics has an action:

$$
S_{V}=\frac{l^{3} N^{2}}{4 \pi e^{4}} \int d^{2} \psi \int e^{3 T}(d T / H)\left[\kappa^{-2}\left(\frac{\partial V}{\partial T}\right)^{2}-\left(\frac{\partial V}{\partial \psi}\right)^{2}\right]
$$

where $e^{4}$ is the coupling constant with $e$ having a dimension of energy, and $\kappa$ is also a constant having a dimension of energy as well.

A good working hypothesis for the constant $\kappa$, which relates the metrics of $\psi$ to the expansion factor $T$, is that $\kappa$ $\sim g^{-1 / 2}$, thus governed by the physics of gravity. On the other hand, the field-field coupling constant $e^{4}$ is likely governed by physics other than gravity, and we shall leave its magnitude as a free parameter.

Moreover, one needs to extend the action $S_{0}$ of Eq. (14) to a more general one:

$$
\begin{aligned}
S= & l^{3} \int e^{3 T}(d T / H)\left[\int d^{2} \psi \sum_{i} \delta\left(\psi-\psi_{i}\right)\right. \\
& \times\left(H^{2} \frac{\left(\psi_{i}^{\prime}\right)^{2}}{2}-\int d^{2} \psi^{\prime} \sum_{j} \delta\left(\psi^{\prime}-\psi_{j}\right) V\left(\psi, \psi^{\prime}\right)\right)+\frac{R}{12 g} \\
& \left.+\frac{\lambda}{2}\left(\frac{n_{f}^{2} \dot{\theta}_{f}^{2}}{\rho_{f}+p_{f}}-\left(p_{f}+\rho_{f}\right)\right)+p_{f}\right]+S_{V},
\end{aligned}
$$

Variation of $S$ with respect to $V$ gives the equation of motion for $V$ :

$$
\frac{e^{-3 T} H}{\kappa^{2}}\left[\frac{\partial}{\partial T}\left(\frac{e^{3 T}}{H}\right) \frac{\partial V}{\partial T}\right]-\frac{\partial^{2} V}{\partial \psi^{2}}=2 \pi \frac{e^{4}}{N} \sum_{j} \delta\left(\psi-\psi_{j}\right) .
$$

Equation (23) shows that there exists a retarded effect in $V$, and hence the functional form $V\left(\left|\psi_{i}-\psi_{j}\right|\right)$, suitable only for static fields, is oversimplified. However, as has been mentioned earlier, each individual field generally has a "nonrelativistic" velocity $\sqrt{g} \psi_{i}^{\prime}$ in the large- $N$ limit and, moreover, $\kappa^{2} \sim g^{-1}$. Therefore, to the leading order of $\sqrt{g} \psi_{i}^{\prime}$, the field $V$ is approximately quasistatic. This is reminiscent of the situation where a collection of slowly moving particles of the same charges generate mostly the electrostatic fluctuations and only little electromagnetic fluctuations. Ignoring the time derivative of $V$ in Eq. (23), we obtain a static, axisymmetric solution for the potential contributed by the discrete source $\psi_{i}$ :

$$
V\left(\left|\psi-\psi_{i}\right|\right)=-\frac{e^{4}}{N} \ln \left|\psi-\psi_{i}\right|
$$

or the Green's function for the two-dimensional Poisson equation.

Finally, we sum up all terms in $S$ explicitly to obtain the total effective action. It follows after an integration by part over $\psi$ that the total action is almost identical to $S_{0}$ of Eq. (18), except for replacing $V_{i, j}$ by $(1 / 2) V_{i, j}$. This factor $1 / 2$ is characteristic of a self-consistent electric or gravitational potential built up from a vacuum. The contribution of interaction potential to the Einstein equations is also given by the new potential, (1/2) $V_{i, j}$, rather than the original one. The above shows that quintessence, its interactions, gravity, and fluid can all be incorporated in a single action.

But, as has been mentioned earlier, the full action with the fluid included has no obvious advantage for algebraic manipulations, and hence we shall from now on remove the fluid action from the quintessence action. Equation (20) will be needed for providing $\rho(T)$ that appears in the quintessence action. Repeating the same procedure, we finally arrive at an explicit form for the quintessence effective action, Eq. (19):

$$
S_{q}=-\int e^{3 T} d T \Gamma^{-1} \sqrt{2 g\left[\frac{e^{4}}{2 N} \sum_{i, j} \ln \left(\left|\psi_{i}-\psi_{j}\right|\right)+\rho_{f}\right]},
$$

from which the equation of motion for each individual field $\psi_{i}$ can be derived.

The above exercise demonstrates that the quintessence potential $V$ can be given a more fundamental origin, and in this particular example we obtain a system of $N$ fields interacting among themselves like a two-dimensional Coulomb gas. If the fields have three components, the interacting system will be like a three-dimensional Coulomb gas. Generally, the $m$-component fields will behave like a $m$-dimensional Coulomb gas. The Coulomb gas self-repels and can maintain a fairly uniform density. It can therefore be a relatively simple $\mathrm{N}$-body dynamical system for the description of the cosmic evolution if the Coulomb gas is initially cold and uniformly distributed. For a finite $N$, the uniformly (in $\psi$ coordinate) distributed fields occupy within a uniform disk of finite area $\pi q^{2}$. The disk experiences a uniform radial force, except at the edge. The outward force at the edge, $-\partial V / \partial q \equiv-|\partial V / \partial \psi|_{|\psi|=q}$, is proportional to $q^{-1}$, characteristic of a two-dimensional Coulomb force. The radius $q$ thus becomes the only relevant dynamical variable for describing the evolution of the field energy density, and we recover the single-field dynamics.

Specifically, Eq. (25) contains $\Gamma^{-1}=\sqrt{1-g \sum_{i}\left(\psi_{i}^{\prime}\right)^{2}}$ and $\sum_{i, j} V_{i, j}$. In a uniform cold disk, the velocity is proportional to the radius $|\psi|$, much like the Hubble expansion, and hence 
$\Sigma_{i}\left(\psi_{i}^{\prime}\right)^{2}=(d \ln q / d T)^{2}\left(2 \pi \int_{0}^{q}|\psi|^{3} d|\psi|\right)\left(N / \pi q^{2}\right)=(N / 2)\left(q^{\prime}\right)^{2}$. On the other hand, the force at the edge can be obtained easily by using the Stokes theorem for a disk of uniform surface density $e^{4} / \pi q^{2}$ : that is, $V(q)=e^{4} \ln (1 / q)+c_{0}$, where $c_{0}$ is a constant serving to offset the ground-state energy. The effective action for the single-field description of this $N$-field problem now becomes

$$
\begin{aligned}
S_{q, e f f}= & -\int e^{3 T} d T\left(1-\frac{N g\left(q^{\prime}\right)^{2}}{2}\right)^{1 / 2} \\
& \times\left\{2 g\left[e^{4} \ln \left(\frac{1}{q}\right)+c_{0}+\rho_{f}\right]\right\}^{1 / 2},
\end{aligned}
$$

or upon being properly normalized,

$$
\begin{aligned}
S_{q, e f f}= & -\int e^{3 T} d T\left[1-g\left(\bar{q}^{\prime}\right)^{2}\right]^{1 / 2} \\
& \times\left\{2 g\left[e^{4} \ln \left(\frac{1}{\bar{q}}\right)+\rho_{f}\right]\right\}^{1 / 2},
\end{aligned}
$$

where $\bar{q} \equiv q \sqrt{N / 2}$ and the constant $c_{0}$ is so chosen that the maximum $\bar{q}$ is unity, a requirement that the initial condition must satisfy. This action describes the $m=2$ case of the Ratra-Peebles potentials [11], $V(q) \propto q^{-(m-2)}$, for the singlefield quintessence, where late inflation can always occur. In fact, all Ratra-Peebles potentials of integer $m-2$ can be generated by this $N$-field model of $m$-component fields.

\section{Warm Coulomb field gas}

The above cold-gas model is of the simplest type. A reasonable extension of it can be for an initial condition where all fields are still uniformly distributed but have thermal velocities. In this case, we need to consider a mean-field phasespace distribution $f\left(\psi, \psi^{\prime}\right)$ to describe the $N$-field kinematics when $N$ is large. That is, while $V(\bar{q})$ remains to be $-e^{4} \ln (\bar{q})$ as is for the cold gas, the quantity $\Sigma_{i}\left(\psi_{i}^{\prime}\right)^{2}$ demands a more elaborate treatment. In the large $N$ limit, the long-range nature of Coulomb interactions allows each field to have a large mean-free path and "sees" only the mean field $V_{m f}$, which equals to $V(\bar{q})$ at $q=\bar{q}$. According to Eq. (3), the distribution function therefore satisfies

$$
\begin{aligned}
\frac{\partial f}{\partial T}+\psi^{\prime} \cdot \frac{\partial f}{\partial \psi}-\Gamma^{-2}\left[\frac{1}{2 g} \frac{\partial \ln V_{m f}}{\partial \psi}\right. \\
\left.-3\left(1-\frac{\rho_{f}+p_{f}}{V_{e f f}}\right) \psi^{\prime}\right] \cdot \frac{\partial f}{\partial \psi^{\prime}}=0 .
\end{aligned}
$$

We follow the mean-field relativistic kinetic theory:

$$
\frac{1}{N} \sum_{i}=\int d^{m} \psi \int d^{m} \psi^{\prime} f\left(\psi, \psi^{\prime}\right)=\int d^{m} \psi n_{b},
$$

where $m$ is the number of field components and the number density $n_{b}$ has been normalized to unity;

$$
\begin{aligned}
\frac{1}{N} \sum_{i} \sqrt{g} \psi_{i}^{\prime} & =\int d^{m} \psi\left[\int d^{m} \psi^{\prime} \sqrt{g} \psi^{\prime} f\left(\psi, \psi^{\prime}\right)\right] \\
& =\int d^{m} \psi\left[n_{b} v_{b}\right],
\end{aligned}
$$

where $v_{b}$ is the local bulk velocity;

$$
\begin{aligned}
\frac{1}{N} \sum_{i} g\left(\psi_{i}^{\prime}\right)^{2} & =\int d^{m} \psi \int d^{m} \psi^{\prime}\left[g\left(\psi^{\prime}\right)^{2} f\left(\psi, \psi^{\prime}\right)\right] \\
& =\int d^{m} \psi\left[n_{b} v_{b}^{2}+2 \frac{U_{\psi}}{N}\right],
\end{aligned}
$$

where $U_{\psi}$ is the thermal energy. Here, we have assumed that $f$ has an isotropic velocity distribution.

The appearance of the thermal energy $U_{\psi}$ adds an extra dynamical variable to this problem, and it needs to be solved self-consistently. This task can be relatively easy to handle when $N$ is large. To a good approximation, each field freely streams, much like an ideal-gas particle, so that the adiabatic ideal-gas law provides the needed description for $U_{\psi}$. For uniformly distributed $N$ fields, we may use a scaling factor $b(t)$ to account for the bulk motion, much like how the scaling factor of the Hubble flow $a(t)$. That is, $\psi_{i}=b(t) x_{i}$ and $\dot{\psi}_{i}=H \psi^{\prime}=\dot{b} x_{i}+b \dot{x}_{i}=H\left(v_{i}+\delta v_{i}\right) / \sqrt{g}$, where $\delta v_{i}$ is the "thermal" velocity. In fact, the individual field $\psi_{i}$ satisfies the original field equation,

$$
\begin{aligned}
0= & \ddot{\psi}_{i}+3 H \dot{\psi}_{i}+\frac{\partial V_{m f}}{\partial \psi_{i}}=\left[\ddot{b}+3 H \dot{b}+\left(V_{m f}^{\prime \prime} / 2\right) b\right] x_{i} \\
& +\left[b \ddot{x}_{i}+\dot{x}_{i}(2 \dot{b}+3 H b)\right] .
\end{aligned}
$$

The first bracket vanishes, as it describes the bulk flow or the uniform expansion. The second bracket must therefore also vanish, thereby allowing $\dot{x}_{i}$ to be solved: $\dot{x}_{i} \propto b^{-2} a^{-3}$. Furthermore, the dynamical variable, the disk radius $q$, is proportional to the field scaling factor $b$, and it thus follows that the thermal energy

$$
U_{\psi}=\left(\frac{1}{2}\right) \sum_{i} \delta v_{i}^{2}=\alpha^{2} q^{-2} a^{-6},
$$

where $\alpha$ is a real constant.

Since $\Sigma_{i} \delta v_{i}^{2}$ has already been solved, there is no need to consider the thermal component of $g\left(\psi_{i}^{\prime}\right)^{2}$ appearing in $H^{2}$ of Eq. (17), and thereafter in $\Gamma$ of the action $S$ in Eqs. (19) and (25). This procedure is identical to how we treated the fluid component in converting $\Gamma$ of Eq. (18) to $\Gamma$ of Eq. (19). Substituting $g \Sigma_{i}\left(\psi_{i}^{\prime}\right)^{2}=\Sigma_{i} v_{i}^{2}+H^{-2} U_{\psi}$ into Eqs. (17), we have the new expressions for the Einstein equations:

$$
\begin{aligned}
H^{2} & =\frac{2 g \bar{V}_{e f f}}{1-N g\left(q^{\prime}\right)^{2} / 2}, \\
\frac{\ddot{a}}{a} & =-2 H^{2}+3 g\left[2 \bar{V}_{e f f}-\left(\rho_{f}+p_{f}\right)\right],
\end{aligned}
$$


where $\bar{V}_{e f f} \equiv V_{m f}(q)+\rho_{f}+U_{\psi}(q, a), \Sigma_{i} v_{i}^{2}=N g\left(q^{\prime}\right)^{2} / 2$. We also have a new expression for the effective quintessence action:

$$
S_{q, e f f}=l^{3} \int e^{3 T} d T \sqrt{2 g \bar{V}_{e f f}\left[1-N g\left(q^{\prime}\right)^{2} / 2\right]} .
$$

Thus, very different from what Eq. (19) or (25) may have suggested, the Lorentz factor here contains only the bulk flow, and the thermal energy contribution is absorbed into $V_{e f f}$. The functional form of $V$ depends on what kind of disk configurations are under consideration. The new effect arising from $U_{\psi}$ will lead to a qualitatively very different result when the potential is attractive, where the balance of the repulsive force of $U_{\psi}$ and the attractive force of $V$ gives rise to quasiequilibrium trajectories, which on one hand rotate on the fast time scale and on the other hand sink to the potential bottom on a secular time scale.

It is of little surprise to find that the effective action of Eq. (35) also describes the dynamics of a single complex field [14], the so-called "spintessence" [15], where we let $N=1$ and $m=2$. The new effect pertaining to $U_{\psi}(q, a)$ is identical to the "centrifugal potential" of "rotational motion" of the single complex quintessence. This is because the "thermal motion" allows the trajectory of each individual field to carry a finite "angular momentum" relative to other fields, and these transverse "velocities" give rise to the "centrifugal forces." However, unlike spintessence, where the $U(1)$ symmetry is globally destroyed to a large degree, the thermal motion of $N$ fields break the $U(1)$ symmetry to a much lesser degree. In particular, when the coarse-grained average of fields of a large $N$ is taken, the $U(1)$ symmetry is nearly restored, at worst broken by $N^{-1 / 2}$ due to the Poisson statistics.

\section{Hamilton-Jacobi equation}

We proceed to discuss how the present formulation can be extended to the Hamilton-Jacobi theory, which will prove to be useful for considerations of the cosmological perturbations of superhorizon size to be discussed later. We shall now return to the general expression, Eq. (18), for the many-field action. Given the Lagrangian in Eq. (18), we find the canonical momenta of the fields and fluid to be

$$
\begin{aligned}
& \pi_{i}=g \bar{\Gamma} \psi_{i}^{\prime} \sqrt{2 V_{e f f}-\rho_{f}-p_{f}} e^{3 T}, \\
& \pi_{f}=g \bar{\Gamma}\left(\frac{n_{f}^{2} \theta_{f}^{\prime}}{\rho_{f}+p_{f}}\right) \sqrt{\left.2 V_{e f f}-\rho_{f}-p_{f}\right)} e^{3 T},
\end{aligned}
$$

where the Lagrangian multipier $\lambda$ has been set to its due value, 1. As the Hamiltonian $h=H e^{3 T}$, it follows from Eq. (17) that

$$
h^{2}=\sum_{i} \pi_{i}^{2}+\left(\frac{\rho_{f}+p_{f}}{n_{f}^{2}}\right) \pi_{f}^{2}+g\left(2 V_{e f f}-\rho_{f}-p_{f}\right) e^{6 T} .
$$

We now employ the Hamilton-Jacobi theory to rewrite this Hamiltonian into

$$
\begin{aligned}
g^{2}\left(\frac{\partial S}{\partial T}\right)^{2}= & \sum_{i}\left(\frac{\partial S}{\partial \psi_{i}}\right)^{2}+\left(\frac{\rho_{f}+p_{f}}{n_{f}^{2}}\right)\left(\frac{\partial S}{\partial \theta_{f}}\right)^{2} \\
& -g\left(2 V_{e f f}-\rho_{f}-p_{f}\right) e^{6 T}
\end{aligned}
$$

where the canonical momenta $\pi_{i}$ and $\pi_{f}$ are cast into $\partial S / \partial \psi_{i}$ and $\partial S / \partial \theta_{f}$, respectively, the Hamiltonian $h \equiv g \partial S / \partial T$, and $S$ is the Hamilton principal function. The solution to this Hamilton-Jacobi equation has the form $S=S_{q}\left(\psi_{i}, T\right)$ $+S_{f}\left(\theta_{f}\right)$, where $S_{q}=W_{q}\left(\psi_{i}, T\right) \exp (3 T)$. The fluid part $S_{f}$ on the right-hand side of Eq. (38) cancels by itself since the time component of the fluid 4-velocity, $n_{f} \dot{\theta}_{f} /\left(\rho_{f}+p_{f}\right)$, equals unity, and it yields $\pi_{f}=\sqrt{g} n_{f} \exp (3 T)$. Furthermore, the fluid momentum $\pi_{f}$ turns out to be a constant since the fluid solution obeys $n_{f} \propto \exp (-3 T)$, thus allowing $S_{f}$ consistently to be independent of $T$ explicitly. Equation (38) then becomes

$$
\sum_{i}\left(\frac{\partial W_{q}}{\partial \psi_{i}}\right)^{2}-g^{2}\left(\frac{\partial W_{q}}{\partial T}+3 W_{q}\right)^{2}=-2 g\left[V+\rho_{f}(T)\right] .
$$

At any given time slice $T=T_{n}$, the phase function $S_{q}$ is a function of $\psi_{i}$, and $\partial W_{q} / \partial \psi_{i}$ is parallel to the velocity $\psi_{i}^{\prime}$. Any virtual displacement $\Delta \psi_{i} \equiv \psi_{i}^{(\alpha)}-\psi_{i}^{(\beta)}$ lying on the $(N m-1)$-dimensional hypersurface $W_{q}\left(\psi_{i}, T_{n}\right)=$ const is perpendicular to the local velocity $\psi_{i}^{\prime}$, where $\psi_{i}^{(\alpha)}$ and $\psi_{i}^{(\beta)}$ are two adjacent instantaneous trajectories at $T=T_{0}$. At the next instant, $T=T_{0}+\delta T$, the hypersurface evolves and so do the two trajectories $\psi_{i}^{(\alpha)}$ and $\psi_{i}^{(\beta)}$. However, at this next instant the two trajectories may not land on the same constant- $W_{q}$ hypersurface. In order for the two instantaneous orbits be on the same hypersurface, they must be evaluated at different times. The progressively asynchronous orbits on the constant- $W_{q}$ hypersurfaces occur regardless whether the fluid is present.

The notion of the constant- $W_{q}$ hypersurface is useful only when $\Delta \psi_{i}$ is sufficiently small, i.e., in the regime of linear perturbations. The reason is that as the multifield dynamics is likely chaotic, $W_{q}$ is generally not an analytical function of $\psi_{i}$. Generally speaking, the constant- $W_{q}$ surface should consist of foliated patches jointed by kinks and cusps, and an analytical $W_{q}$ exists only in some immediate neighborhoods of a given location $\psi_{i}$. Hence, as long as $\Delta \psi_{i}$ is small, two trajectories can always stay in the same foliation patches at different times. In the next section, we will make use of this notion to discuss the cosmological linear perturbations in the comoving gauge.

\section{COSMOLOGICAL PERTURBATIONS}

The above formulation using $T(\equiv \ln a)$ as the time variable is a natural choice for describing the coupled dynamics of the background scalar field and gravity. It turns out that 
such a choice is also convenient for analyzing the cosmological perturbations of space curvature [10].

In the very long-wavelength limit, for which the wavelength $k^{-1}$ is much greater than the Hubble radius $H^{-1}$, the quintessence and matter perturbations, $\delta \psi_{i}$ and $\delta \rho_{f}$, can be regarded as spatially homogeneous. The energy-momentum tensor of each matter component is diagonal in the limit $k / H \rightarrow 0$, since the off-diagonal component involves two spatial derivatives and hence is of order $(k / H)^{2}$ and the spacetime component is of order $(k / H)$. Such a spatially homogeneous diagonal energy-momentum tensor leads to diagonal metrics. Thus, superposed with superhorizon perturbations of $k / H \rightarrow 0$, both the scalar field and fluid satisfy the same equations of motion as those of the background derived in Sec. III A. The cosmological perturbations are therefore the deviation of different trajectories of slightly different initial conditions.

In fact, one can do better than the above zeroth order (of $k / H)$ considerations and retain the perturbed quantities of order $k / H$. It is well known that the metric fluctuations have gauge degrees of freedom, and some gauge choices may be more convenient than others. As has been shown by Sasaki and Tanaka [10], the gauge that keeps the time variable $T$ unperturbed turns out to be the convenient gauge in allowing one to calculate perturbations of order $k / H$, specifically for the perturbed space curvature $R$ and the traceless stress. In this gauge, the perturbed shift (space-time component) of the metrics vanishes and the perturbed space compression (trace of space component) of metrics remains a constant. The equation of motion derived above, describing the " 00 " component and the trace of the space components of the energymomentum tensor, remains valid even up to $O(k / H)$ and is decoupled from other tensor components of size $(k / H)$, namely, the perturbed space curvature and traceless stress. The great advantage of this gauge over, for example, the synchronous gauge is that the perturbed space curvature can be directly determined from the freely decaying traceless stress.

Although Sasaki and Tanaka's work does not include the fluid component, the form of the metrics remains the same as the pure-scalar field case when the fluid is present. One can show that the leading-order contribution from the fluid in this constant $T$ gauge is from the 00 component and the trace of space components of the perturbed fluid energy-momentum tensor. Moreover, as the perturbed $\delta \rho_{f}$ and $\delta p_{f}$ are related by the underlying equation of state, $\delta \rho_{f}$ is simply $c_{0}\left(\rho_{f}+p_{f}\right)$ with a constant $c_{0}$. In addition, the perturbed time component of the fluid 4-velocity $\delta U_{0}$ is simply $-\delta H / H$, the perturbed Hubble parameter; the space component $\delta U_{i}$ appears only on the order of $O(k / H)$ in the $0 i$ component of the perturbed energy-momentum tensor in the same manner as the scalar fields do. Hence, inclusion of the fluid retains all the good features of the constant- $T$ gauge discussed by Sasaki and Tanaka for pure scalar fields. This is due partly to that the fluid dynamics can be formulated in a way very similar to that of the scalar field, as has been shown in Sec. III A, and partly to that the formulation using $T$ as a new time variable has already absorbed the gravity in the matter dynamics in a self-consistent manner.
Since the cosmological perturbations result from the departure of slightly different initial conditions, there exist $2(\mathrm{Nm}+1)$ independent perturbations, due to the $2(\mathrm{Nm}$ $+1)$ independent phase-space coordinates for the $N$ scalar fields of $m$ components, plus a fluid component. After one manages to make the $2(\mathrm{Nm}+1)$ scalar modes orthogonal, the space curvature of the constant- $T$ hypersurface only exists in two canonically conjugated modes, and vanishes in all others. These two modes are the adiabatic growing and decaying modes, and the rest are entropy modes. The growing adiabatic mode is associated with two trajectories with exactly the same initial phase-space coordinates but differing by a $\Delta T_{0}$ in their initial launching times. The two orbits follow exactly the same phase-space trajectories, but at any given time $T$ their phase-space coordinates are different. This time-translational mode exists regardless of whether the fluid is present.

Among all independent perturbations, some may be integrable and thus stable, some are chaotic and thus unstable, and the rest can still follow some stable manifolds in the phase space and thus be stable. Note that the time-translation mode is always stable. For those perturbations that are integrable, they are all associated with some integrals of motion $\alpha_{j}$ 's, and result from different trajectories with slightly different $\alpha_{j}$ 's. For example, the above Coulomb field gas has a conserved total angular momentum, $L$, and there exists an integrable angular-momentum perturbation. Since the Coulomb gas repels, the "rotational motion" must slow down and hence the energy density of the angular-momentum mode decays in time. For those that follow stable orbital manifolds, these modes eventually converge into some attractors since this dissipative nonlinear system has attractors in various regions of the $2 \mathrm{Nm}$-dimensional phase space. These modes are usually associated with the "velocity" degree of freedom, as the presence of cosmic drag makes velocities eventually die out. By contrast, the chaotic unstable modes are usually associated with the "position" degree of freedom since the initial transient can render the orbits to end up at very different locations; these chaotic unstable modes give rise to perturbations in the potential energy $V_{\text {eff }}$. In great contrast to the single-field case, there are many independent entropy modes in the multifield system, and many of these entropy modes can be more unstable than the adiabatic fluctuations.

As the multifield dynamics is likely chaotic, the perturbations tend to be entangled. Though the constant- $T$ slice provides a convenient way to calculate the cosmological perturbations, the physically relevant time slice is such that the perturbed energy-momentum tensor has a vanishing $0 i$ component, i.e., the comoving slice. This is due to the fact that the constant- $T$ gauge contains an extra gauge mode, which is always attached to the time-translational mode mentioned earlier. This gauge mode can be removed in the comoving gauge.

The matter rest frame requires $T_{0}^{i}=0$, or

$$
k\left[\sum_{i} \Delta \psi_{i} \psi_{i}^{\prime}+\Delta \theta_{f} \theta_{f}^{\prime}\left(\frac{n_{f}^{2}}{\rho_{f}+p_{f}}\right)\right]=0,
$$


where $k$ is the small but finite wave number of the space Fourier mode. It means that the difference of two adjacent trajectories is constrained to be perpendicular to the background "momentum" [cf. Eq. (36)]. As $\Delta \psi_{i}$ and $\Delta \theta_{f}$ lie on an $\mathrm{Nm}$-dimensional comoving hypersurface in the $(\mathrm{Nm}$ $+1)$-dimensional embedded space, this $\mathrm{Nm}$-dimensional comoving time slice is simply the $\left.S\left(\psi_{i}, \theta_{f}\right)\right|_{T}=$ const hypersurface, where $S$ is the Hamilton principal function.

We recall the discussions at the end of Sec. III D that two adjacent orbits must be evaluated at different times $\Delta T$ for them to lie on the same constant- $W_{q}$ hypersurface. Now, the arguments should be extended to include the fluid, and $W_{q}$ is replaced by $S$. The difference of nearby trajectories $\delta \psi$ and $\delta \theta_{f}$ at the constant- $S$ surface therefore satisfies

$$
\begin{aligned}
\sum_{i} \psi_{i}^{\prime}\left[\delta \psi_{i}(T)+\psi_{i}^{\prime} \Delta T\right] \\
+\left(\frac{n_{f}^{2}}{\rho_{f}+p_{f}}\right) \theta_{f}^{\prime}\left[\delta \theta_{f}(T)+\theta_{f}^{\prime} \Delta T\right]=0,
\end{aligned}
$$

thereby giving the time difference

$$
\Delta T=-\frac{\sum_{i}\left(\delta \psi_{i} \psi_{i}^{\prime}\right)+\left(n_{f}^{2} / \rho_{f}+p_{f}\right) \delta \theta_{f} \theta_{f}^{\prime}}{\sum_{i}\left(\psi_{i}^{\prime}\right)^{2}+\left(n_{f}^{2} / \rho_{f}+p_{f}\right)\left(\theta_{f}^{\prime}\right)^{2}},
$$

where $\delta \psi$ and $\delta \theta_{f}$ are the difference of two adjacent orbits evaluated at the same $T$. For the time translational mode, we have vanishing $\delta \phi_{i}$ and $\delta \theta_{f}$ when observed in the comoving slice, and hence $\Delta T_{0}=\delta \psi_{i} / \psi_{i}^{\prime}=\delta \theta_{f} / \theta_{f}^{\prime}$, thereby yielding $\Delta T=-\Delta T_{0}$. This cancels the original enhancement of curvature perturbation $R$ due to the difference of initial launching times $\Delta T_{0}$ in the constant- $T$ gauge, and reverts the curvature perturbation back to its intrinsic value $R_{0}$ given by initial quantum fluctuations. That is, the contribution of $\Delta T_{0}$ to the perturbed space curvature $R$ in this constant- $T$ gauge is the gauge degree of freedom and removed in the comoving gauge $[10,16]$.

\section{CONCLUSIONS AND DISCUSSIONS}

We formulate the coupled dynamics of quintessence, gravity, and fluid in a unified framework by using the logarithmic cosmic expansion factor $T(\equiv \ln a)$ as the new time variable. The field dynamics turns out to be identical to the dynamics of a relativistic particle immersed in a static viscous medium. The presence of a fluid component serves merely to weaken the force acting on the field. Such formulation can be extended to describing many-field dynamics as a many-body problem. The forces on these fields can be made to be self-consistent ones by incorporating the mediate scalar bosons in the field functional space. This work also points out that when the number of fields is large, it is possible to reduce the many-field problem to an effective singlefield problem. We work out the cold Coulomb field gas and warm Coulomb field gas as two examples.
It is interesting to note that when the multifield dynamics is cast in the framework of relativistic Hamilton-Jacobi theory, it can be straightforwardly extended to quantum mechanics where Eq. (38) becomes the Klein-Gordon equation with an evolving squared mass, $2 g V_{e f f} e^{6 T}$. This can be made exact when $\rho_{f}=0$ in the very early universe before photons were produced. The quantization is with respect to the scalar field $\psi_{i}$ relative to the conjugate momentum $\pi_{i}$ [cf. Eq. (36)], as well as the Hamiltonian $h$ relative to the conjugated expansion factor $\ln a(=T)$ [cf, Eq. (37)]. Note that the gravity has been incorporated in the quantization since the action of Eq. (18) contains the gravity piece. Due to the presence of a time-dependent squared mass in the Klein-Gordon equation, it gives rise to low-energy $(\sim \hbar H)$ quantum fluctuations of the quintessence field and metrics on the superhorizon scale.

The quantization of $\psi_{i}$ in the field space logically fits well with our earlier treatment of the scalar bosons $V$, which mediate the quintessence interactions discussed in Sec. III, in the field functional space. (Of course, quantization of the scalar bosons $V$ can also be performed when necessary.) As has been pointed out immediately below Eqs. (7) and (8), the ultrarelativistic regime renders the Hamiltonian $h$ to be a good constant of motion. In this regime, the interactions $V_{i, j}$ are comparatively small, and the dominant energy component is the free field. It thus provides an easy way for quantization of $\psi_{i}$ together with the gravity along the light cone.

The space coordinates have been dropped out of the scene in the above analyses because we are confined to the superhorizon scales. It may be regarded as a particular reference frame where the dynamics takes place in the rest frame of the space coordinates (in contrast to the field coordinates) with a vanishing space momentum. It remains to be investigated whether the Planck-scale physics really admits such a symmetry that one can generally transform the reference frame to the proper frame where the space momentum vanishes. For example, when the uncertainty principle applies to the classical notions of space and time in the Planck era, space can become fuzzy in a reference frame where time has a definite value. If so, the Bose-Einstein condensation of a collection of $N$ such superhorizon quanta may constitute the foundation for the background $N$ quintessence fields as well as the gravity in the Planck scale, and provide the needed energy content to develop into one universe.

We now return to discussions on the classical quintessence fields. Clearly, the dynamics of classical multifield can easily be nonintegrable and chaotic. Though a large number of fields help simplify the problem, such a step is probably too drastic to take for a possible extension beyond the singlefield quintessence. It is probably more natural to investigate dynamics of a few coupled fields. However, this is surely a difficult regime as far as the chaotic dynamics is concerned, since the coarse-grained averaged quantities do not exist. Nevertheless, since the friction applies uniformly in the velocity space, the acceleration may quickly become negligible and the velocities become non-relativistic after an initial transient, especially when the fluid energy density is nonnegligible and weakens the forces on the quintessence fields. In such a "rolling" regime, half the degrees of freedom are 
suppressed and the dynamics becomes much simpler. However, even in the presence of such suppression of freedom, if the remaining degrees of freedom exceed or equal 3 , it is possible that there exist strange attractors, where trajectories can stretch, twist, and fold in a multidimensional manifold.

In passing, we would like to point out that the Bianchi type IX, mix-master model, in which the universe is homogeneous but anisotropic and the world geodesics is described by three-dimensional rotation [17], can in fact be cast into a two-field quintessence model. The two anisotropic degrees of freedom in the mix-master model correspond to two quintessence fields, and the space curvature $R^{(3)}$, which is explicitly time dependent, plays the role of $-V\left(\phi_{i}\right)$. This model had been under intense studies in the 1970s, and the foci were placed on the dynamics near the initial singularity. The dynamics of the mix-master model is known to be chaotic due to the presence of regions in $V\left(\phi_{i}\right)$ where the space curvature is negative.

The chaotic scalar-field dynamics in the early universe can have imprints in the late-time cosmological perturbations. Depending on the nature of the multifields, entropy perturbations may have larger field amplitudes than the adiabatic perturbations do if indeed multifields were active in the early universe. They generally yield a different fluctuation power spectrum in the cosmic microwave background radiation (CMBR) from the one given by the pure adiabatic perturbation. The entropy modes, when generated during the inflation, normally lead to a spectrum of temperature perturbations dominated on the large scale. To be consistent with the observed CMB temperature anisotropy spectrum, $k^{n}$ with $n \approx 1$ over three orders of magnitude in length scale and the COBE normalization on the large scale [18], the entropy modes cannot be too unstable during the inflationary epoch. Physically, it is a natural outcome of inflation, since the po- tential $V(\phi)$ is flat during inflation and the field dynamics is slow, therefore inhibiting evolution in the cosmological perturbations. Nevertheless, the entropy mode may become unstable toward the end of, or after, the inflation. Instabilities at a later time give rise to smaller-scale power in the CMB fluctuations, and the smaller-scale fluctuations play a crucial role for structure formation. In view of the relatively large error bars in the CMB measurements of fluctuation spectrum at angular scale $l>600$ [4-6], there can still be a sizable range of the astrophysically relevant length scale for accommodating the entropy modes resulting from multifield quintessence in the early universe.

Moreover, the chaotic dynamics of multifield quintessence may also serve as a solution to a problem currently facing the string thoery. A universe with accelerating expansion presents a future horizon, making the scattering theory of particle interactions, on which the $M$-theory is based, become ill-defined [19]. Without fine-tuning, a single-field quintessence often makes the universe expansion accelerate forever once it starts [20]; however, the multifield quintessence may instead naturally yield a universe that the accelerating expansion proceeds only temporarily due to the chaotic dynamics.

Finally, it should be mentioned that though we have suggested, in Sec. III, the interacting potential $V\left(\phi_{i}\right)$ of the multifield quintessence to arise from interacting bosons in the field space, other possibilities have also been proposed recently. They are motivated either by the supergravity or string theory [20], the supersymmetric QCD [21], or by obtaining a small but nonvanishing cosmological constant [22]. These interacting potentials should all be constrained by the phenomelonogical issues, such as nucleosynthesis [23], a consideration that is not yet addressed in this paper.
[1] S. Perlmutter et al., Nature (London) 391, 51 (1998); Astrophys. J. 517, 565 (1999).

[2] A.G. Riess et al., Astron. J. 116, 1009 (1998).

[3] A.G. Riess et al., Astrophys. J. 560, 49 (2001); M.S. Turner and A.G. Riess, astro-ph/0106051; S. Jha et al., astro-ph/00101521.

[4] Boomerang Collaboration, P. de Bernardis et al., Nature (London) 404, 955 (2000).

[5] Maxima Collaboration, S. Hanany et al., Astrophys. J. Lett. 545, L1-L4 (2000).

[6] C. Pryke, N.W. Halverson, E.M. Leitch, J. Kovac, J.E. Carlstrom, W.L. Holzapfel, and M. Dragovan, astro-ph/0104490.

[7] R.R. Caldwell, R. Dave, and P.J. Steinhardt, Phys. Rev. Lett. 80, 1582 (1998); C. Wetterich, Nucl. Phys. B302, 668 (1988); I. Zlatev, L. Wang, and P.J. Steinhardt, Phys. Rev. Lett. 82, 896 (1999); V. Faraoni, Phys. Rev. D 62, 023504 (2000); W. Zimdahl, D.J. Schwarz, A.B. Balakin, and D. Pavon, ibid. 64, 063501 (2001); L. Amendola, ibid. 62, 043511 (2000); L. Amendola and D. Tocchini-Valentini, ibid. 64, 043509 (2001); J.D. Barrow, R. Bean, and J. Magueijo, Mon. Not. R. Astron. Soc. 316, L41 (2000); J.P. Uzan, Phys. Rev. D 59, 123510
(1999); T. Chiba, ibid. 60, 083508 (1999); O. Bertolami and P.J. Martins, ibid. 61, 064007 (2000).

[8] J.H. Traschen and R.H. Brandenberger, Phys. Rev. D 42, 2491 (1990).

[9] L. Kofman, A. Linde, and A.A. Starobinsky, Phys. Rev. Lett. 73, 3195 (1994); Phys. Rev. D 56, 3258 (1997); B. Ovrut and P.J. Steinhardt, Phys. Rev. Lett. 53, 732 (1984); O. Bertolami and G.G. Ross, Phys. Lett. B 171, 163 (1986).

[10] M. Sasaki and T. Tanaka, Prog. Theor. Phys. 99, 763 (1998).

[11] B. Ratra and P.J.E. Peebles, Phys. Rev. D 37, 3406 (1988).

[12] T. Chiueh, Phys. Rev. E 49, 1269 (1994).

[13] See, for example, J.D. Jackson, Classical Electrodynamics, 2nd ed. (Wiley, New York, 1975), p. 580.

[14] J.A. Gu and W-Y.P. Hwang, Phys. Lett. B 517, 1 (2001).

[15] L.A. Boyle, R.R. Caldwell, and M. Kamionkowski, astro-ph/0105318.

[16] M. Sasaki and E.D. Stewart, Prog. Theor. Phys. 96, 71 (1996); H. Kodama and T. Hamazaki, Phys. Rev. D 57, 7177 (1998).

[17] C.W. Misner, Phys. Rev. Lett. 22, 1071 (1969); V.A. Belinsky and I.M. Khalatnikov, Sov. Phys. JETP 32, 169 (1970).

[18] R. Strompor, K.M. Gorski, and A.J. Banday, Astrophys. J. 463, 8 (1996). 
[19] S. Hellerman et al., J. High Energy Phys. 06, 003 (2001); W. Fischler et al., ibid. 07, 003 (2001); E. Witten, hep-th/0106109.

[20] T. Chiueh and X.G. He, Phys. Rev. D (to be published), astro-ph/0107453.
[21] M.C. Bento, O. Bertolami, and N.C. Santos, Phys. Rev. D 65, 067301 (2002).

[22] Y. Fujii, Phys. Rev. D 62, 064004 (2000).

[23] A. Masiero, M. Pietroni, and F. Rosati, Phys. Rev. D 61, 023504 (2000). 